\title{
Fingering Instability in Combustion
}

\author{
O. Zik ${ }^{(1)}$, Z. Olami ${ }^{(2)}$ and E. Moses ${ }^{(1)}$ \\ ${ }^{(1)}$ Department of Physics of Complex Systems and ${ }^{(2)}$ Department of Chemical Physics, \\ The Weizmann Institute of Science, Rehovot 76100, Israel
}

\begin{abstract}
A thin solid (e.g., paper), burning against an oxidizing wind, develops a fingering instability with two decoupled length scales. The spacing between fingers is determined by the Péclet number (ratio between advection and diffusion). The finger width is determined by the degree two dimensionality. Dense fingers develop by recurrent tip splitting. The effect is observed when vertical mass transport (due to gravity) is suppressed. The experimental results quantitatively verify a model based on diffusion limited transport.
\end{abstract}

Harnessing combustion is a basic element of technology, from lighting a candle [1] to propelling a spacecraft. The transition to unrestrained conflagrations is often determined by fundamental issues regarding the stability of combustion fronts. Although characterized by tens of concurrent chemical reactions which depend exponentially on temperature [2], these complex systems are governed by the availability of three basic constituents: oxidant, fuel and heat. The experimental control of these coupled fields can be considerably improved by studying the slow combustion regime termed smoldering [2] [ The evolution of a smoldering front is of particular importance for fire safety, since the initial development of a slow burning process will determine whether the fire grows or extinguishes.

The stability of one dimensional fronts has been extensively studied in simpler, less reactive, growth systems [4]. In these systems the pattern is typically characterized by one length scale (the fastest growing wavelength in the linear spectrum). Notable examples are solidification [5], liquid-crystals [6] and viscous fronts [7]. Interfacial instabilities are also known in combustion. The most studied is the thermal-diffusion instability in 'premixed' flames (where the oxygen and gaseous fuel are mixed before they react). This instability results from the competition between the destabilizing effect of molecular transport and the stabilizing effect of heat transport [2], [8], [9]. Previous studies of flame propagation over thin solid fuels focused mainly on flame spread rate [10], [11]. A cellular structure observed in this system was recently related to the thermal-diffusion instability [12].

In this paper we report a new effect: a combustion front in a quasi two dimensional geometry, exhibits a directional fingering instability with two decoupled length scales (the finger width and the spacing between fingers). The non-dimensional control parameter is the Péclet number $P e$ which measures the relative importance of molecular advection and diffusion [13]. The characteristic scale (finger width) is effectively independent of $P e$. It is determined by the degree of two dimensionality. On the other hand, the distance between fingers is self consistently determined by $P e$. The fingering effect has independently been 
observed in a recent experiment conducted in space [14]. Our measurements are performed in the experimentally preferable smoldering regime, where the front can be reproducibly controlled. The oxidizing gas is supplied in a uniform flow, opposite to the direction of the front propagation (i.e., the fuel and oxygen are fed to the reaction from the same side). This configuration enhances the destabilizing effect of reactant transport, which plays the same role as in the thermal-diffusion instability. Although heat transport plays a key role in the instability, our phenomenological model shows that reactant transport alone determines the front velocity and spacing between fingers. The measurements are in remarkable agreement with this model (no free parameters).

The setup is shown schematically in Fig. 1. The experiment is initiated by igniting a thin fuel sample along a line at its downstream edge. The main experimental controls are the flow velocity of oxygen $V_{O 2}$ and the vertical gap between plates $h$. The 'fuel' is filter paper. However, the effect is fuel independent. It occurs in all the combustible materials that we examined, e.g., standard stationery paper, cellulose dialysis bag and polyethylene sheets. In many of these materials the front involves melting and a glowing flame which complicates the measurements.

The front is smooth when the oxygen flow velocity $V_{O 2}$ is higher than some critical value (Fig. 2 2a). When $V_{O 2}$ is slightly decreased the front develops a structure which marks the onset of instability (Fig. $2 b$ ). Further decreasing $V_{O 2}$ yields a periodic cellular structure or 'cusps' (Fig. 2 2 c). As $V_{O 2}$ is gradually decreased, the cusps become sharper until the peaks separate and a fingering pattern is formed. A typical fingering pattern is shown in Fig. 22d. It develops by recurrent tip splitting. Fingers that are closer to the oxygen source screen neighboring fingers from the source of oxygen. The screened fingers stop growing and the tips of the screening fingers split. The splitting is such that both the average finger width and the spacing between the fingers remain unchanged. When $V_{O 2}$ is further decreased the spacing between fingers increases and we obtain sparse fingers with no tip splitting (Fig. 22e). Combustion occurs only in the limited vicinity of the tip, there is no reaction behind the front (along the fingers). A bright spot (like the one in the central finger in Fig. 2 $2 e$ ) signals a local transition from 'smoldering' to 'flame', which typically appears just before a tip stops growing. Fig. $2 c-e$ shows the existence of two well-defined length scales in the fingering pattern, the average finger width $w$ and the average spacing between fingers $d$. While $w$ depends very weakly on $V_{O 2}, d$ is a rapidly decreasing function of it. The maximal value of $d$ is comparable to the system size $d \sim L$. The minimal value of $d$ (zero) is reached when the increase in oxygen flow wipes out the pattern (Fig. $2 c$ ). Tip splitting is observed at $d \sim w$.

The above observations are explained in terms of the following phenomenological picture. At high values of $V_{O 2}$ the uniformly fed front is smooth. As the supply is decreased, small bumps that exist along the interface begin to compete over the oxygen. This mechanism resemble the thermal diffusive instability [2]. The consumption of oxygen drive lateral diffusion currents. When the oxygen supply is further decreased, the bumps consume all the oxygen that is available in their vicinity, and separate into distinct fingers. The 'upstream' fingers (which are closer to the oxygen source) prevail and then tip-split to maintain the same average $d$. As the oxygen supply is further decreased $d$ grows. At a certain stage $d$ is sufficiently large to allow fingering without screening (and tip splitting). Unlike $d, w$ is independent of oxygen supply. It depends on heat transport which is neglected in the above 
simplified picture.

The two dominant oxygen transport mechanisms are diffusion and advection. In the quasi $2 d$ configuration, the Péclet number is $P e=\frac{V_{\mathrm{O}_{2}} h}{D}$ [15]. Dimensional analysis leads us to expect that the measured quantities $u, d$ and $w$ depend on the dimensional parameters $h, V_{O 2}$ and $D$ and on $P e$. Thus, we non-dimensionalize $\tilde{w}=\frac{w}{h}, \tilde{d}=\frac{d}{h}$ and $\tilde{u}=\frac{u}{V_{O 2}}$.

In the oxygen limited case expect all the available oxygen to be consumed and write a mass conservation equation (see the reference in [3]):

$$
\tilde{u} \frac{\tilde{w}}{\tilde{w}+\tilde{d}}=A
$$

where the constant $A$, defined in [16], represents the stoichiometry. $\frac{w}{w+d}$ is the fraction of burned area, resulting from integration over the width of the sample $L$. The deficiency of oxygen produces concentration gradients and consequently lateral diffusion currents (see Fig. 3.), given by $j_{x}=D \nabla_{x} \rho_{0_{2}}$, where $\rho_{0_{2}}$ is the oxygen concentration. The gradient is typically over a distance of $d+w$. At steady state, the lateral current satisfies $j_{x}=u \rho_{02}$ up to a proportionality constant of order unity. This yields an equation for the velocity of the diffusion limited growth [17]

$$
\tilde{u}=\frac{1}{(\tilde{w}+\tilde{d})} P e^{-1}
$$

Combining Eq. 1 and Eq. 2 gives

$$
\begin{array}{r}
\tilde{d}=\sqrt{\frac{\tilde{w}}{A}} P e^{-\frac{1}{2}}-\tilde{w} \\
\tilde{u}=\sqrt{\frac{A}{\tilde{w}}} P e^{-\frac{1}{2}}
\end{array}
$$

For $P e$ greater than some critical value $P e_{c}$ we observe a flat front (Fig. 2). For $P e$ smaller than another critical value $P e_{c 1}$ we observe fingers. We regard the narrow band $P e_{c 1} \leq P e \leq P e_{c}$ as onset regime. In this regime we observe a connected front with a cellular structure. Eq. 3 yields the critical $P e_{c 1}$ for the onset of a connected front, when $d$ goes to zero:

$$
P e_{c 1}=\frac{1}{A \tilde{w}}
$$

The mass conservation equation (Eq. 1) holds for $P e \leq P e_{c}$. In dimensional units, it predicts a linear dependence of the quantity of burned material per unit time $u w /(w+d)$ on $V_{O 2}$. This dependence is shown in Fig. \#. The measured slope is $A=0.043 \pm 0.005$. The predicted slope is $A=0.051 \pm 0.005$ [16]. The numerical agreement (within experimental error) shows that the system is well within the oxygen deficient regime. The front velocity $u$ is shown in the insert. Below a critical value corresponding to $P e_{c 1}$ (arrow in the figure), it behaves as $u \sim V_{O 2}{ }^{\frac{1}{2}}$ as predicted by Eq. 4. At $P e_{c 1}$ there is a crossover to the linear dependence predicted by Eq. 1 with $d=0$. 
The dependence of the lengths $d$ and $w$ on $P e$ is shown in Fig. 5. The ratio between $w$ and $d$ characterizes the pattern. It is determined by $P e$. Sparse fingers are observed at $\frac{d}{w}>>1(0.1 \leq P e \leq 2.6)$, tip splitting at $\frac{1}{4} \leq \frac{d}{w} \leq \frac{5}{4}\left(2.6<P e \leq P e_{c 1}\right)$. Experimentally $P e_{c 1}=17 \pm 1$. The predicted value is $P e_{c 1}=1 / A w \approx 21$ (Eq. 5). A connected front is observed at $P e_{c 1} \leq P e \leq P e_{c}$. In this regime $d=0$ and $w$ is the characteristic 'cusp' size. Experimentally $P e_{c}=22 \pm 1$ [18]. $w$ and $d$ are not defined for $P e \geq P e_{c}$. The continuous line in Fig. 5 shows the theoretical prediction (Eq. 3). It is in very good agreement with the data points. These measurements were performed by varying $V_{O 2}$ at $h=0.5 \mathrm{~cm}$. Other values of $h$ (in the range $0.2 \mathrm{~cm} \leq h \leq 1 \mathrm{~cm}$ ) show the same qualitative dependence of $d$ on $P e$. We conjecture that the slow linear increase of $w$ as a function of $P e$ is related to the limitation posed by the reaction rate 15].

The lengths $w$ and $d$ are weakly coupled via Eq. 3. Fig. 6 exemplifies an experiment in which we isolated $w$ from $d$ in two narrow pieces of paper $(L=1 \mathrm{~cm}$ and $L=0.6 \mathrm{~cm})$. Both fronts propagated at the same velocity $u$ and with the same $w$ of the main trunk, as in the wide system (within experimental scatter). At $L=1 \mathrm{~cm}$ we observe periodic attempt to tip split which can not be accomplished (since $L<w+d$ ). We conclude that the instability is not a collective effect but a feature of the single finger. The competition over oxygen determines $d$ but not $w$.

The weak dependence of $w$ on $P e$ allows us to measure $w$ as a function of $h$ in a wide range of $P e$. Fig. 7 shows that $w$ is dominated by the degree of two-dimensionality $h$ [18. We conjecture that the origin of this dependence is related to heat transport, via convection of combustion products. This conjecture is consistent with our observation that $w$ is a decreasing function of the fraction of cooling $\left(N_{2}\right)$ gas in the feeding mixture. The linear dependence of the characteristic length of the instability on the spacing between plates is known to occur in this ('Hele Shaw') geometry in viscous fronts [4]. To some extent, $w$ also depends on the features of the fuel, heat conductivity of the bottom plate and the relative nitrogen concentration in the feeding gas.

In conclusion, the complex burning process exhibits a rich but controllable pattern. A flat piece of fuel (e.g. paper), forced to burn against an oxidizing wind, develops a steady fingering state. This new effect occurs when vertical flow is suppressed. There are two key processes, transport of heat and transport of reactants, and two effectively decoupled length scales: the finger width and the spacing between fingers. The characteristic length (finger width) is very weakly dependent on the control parameter (Péclet number). It is determined by the geometry (degree of two dimensionality). The spacing between fingers is determined by the Péclet number. This dependence is in very good quantitative agreement with a phenomenological model which we base on reactant transport. Significant effort must be devoted to the onset regime, where preliminary observations already revealed interesting time-dependent modes. 


\section{REFERENCES}

[1] M. Faraday, The Chemical History of a Candle, W. Croookes (Chato Windus, London, 1882).

[2] G.I. Sivashinsky, Ann. Rev. Fluid Mech. 15, 179 (1983).

[3] Smoldering is a non-flaming mode of combustion (i.e., the emitted gas does not 'glow' in the visible light) where oxygen interacts with a solid fuel to produce char, gaseous products and the heat that sustains the process. See: A.C. Fernandez-Pello, B.J. Matkowsky, D.A. Schult, and V.A. Volpert, Combust. and Flame 101, 471 (1995).

[4] P. Pelce, Dynamics of Curved Fronts (Academic Press, 1988).

J. Krug and H. Spohn, Solids Far From Equilibrium (Cambridge University Press, Cambridge, 1992).

A.-L. Barabasi, H. E. Stanley, Fractal concepts in surface growth (Cambridge University Press, 1995).

T. Halpin-Healey and Y.-C. Zhang, Physics Reports, 254, 215 (1995).

Y. Couder in Chaos, Order and Patterns R. Artuso, P. Cvitanovic, G. Casati, Eds. (Plenum Press, New York, 1991) p. 203.

[5] J. S. Langer, Rev. Mod. Phys. 52, 1 (1980).

[6] J. M. Flesseles, A. J. Simon and A. J. Libchaber, Advances in Physics 40, 1 (1991).

[7] P.G. Saffman and G.I. Taylor, Proc. Roy. Soc. 245,155 (1958).

[8] P. Pelce and P. Clavin, J. Fluid Mech. 124, 219 (1982).

[9] M. Gorman, M. el-Hamdi, B. Pearson and K.A. Robins, Phys. Rev. Lett. 76, 228 (1996).

[10] J. de-Ris, Twelfth Symposium on Combustion, Combustion Institute, Pittsburgh (1969).

A. E. Frey and J. S. Tien, Combust. Flame 36, 263 (1979).

A. E. Frey and J. S. Tien, Combust. Flame 26, 257 (1976).

[11] S. L. Olson, Combust. Sci. and Tech. 26, 76 (1991)

[12] Y. Zhang, P.D. Ronney, E.V. Roegner and J.B. Greenberg, Combust. and Flame 90, 71 (1992).

[13] For the definition of $P e$ see: L.D. Landau and E.M. Lifshitz, Fluid Mechanics 209 (Pergmanon Press, London, 1976). For a discussion on the role of $P e$ in smoldering, see: J. Buckmaster, IMA J. Appl. Math. 56, 87 (1996).

[14] The reduction to two dimensions is aimed to suppress the effect of convection and plumes near the front. A $3 d$ experiment akin to ours has been recently conducted onboard the space-shuttle (see: T. Kashiwagi and S. L. Olson, Radiative Ignition and Transition to Spread Investigation, Proceeding of USML-2/USML-3, National Academy of Science (1997)). The similarity of the results presents a remarkable demonstration of the equivalence of quasi $2 d$ and micro-gravity in neutralizing gravitational effects. The micro-gravity data of Kashiwagi and Olson has the same qualitative dependence of the lengths and front velocity on oxygen supply, as in our two dimensional results.

[15] $P e$ accounts for the limitation imposed by diffusion. Another limiting factor is the chemical reaction. The corresponding non-dimensional parameter is the Damk" ohler number $D a$ defined as the ratio between the chemical reaction rate to the molecular transport rate (see: R. A. Strelow, Combustion Fundamentals, (McGraw-Hill, Singapore, 1985)). In our experiment $D a$ is of order unity. However, the developed state is diffusion limited and $D a$ can be neglected in the current discussion.

[16] The stoichiometric factor is $A=\frac{a \rho_{O 2}\left(h-\tau_{u b}\right)}{\mu\left(\rho_{u b} \tau_{u b}-\rho_{b} \tau_{b}\right)}$, where $\mu$ is the stoichiometric coefficient, 
defined as the mass of reactant gas consumed by reacting with one unit mass of solid. $u$ is the front velocity. $\rho_{\mathrm{O} 2}$ is the oxygen density, $a$ is the fraction of oxygen in the gas mixture. $\tau$ is the fuel thickness. The subscripts $b$ and $u b$ stand for burned and unburned solid respectively. To calculate the slope in Fig. 1 we used $\mu=1.18$ (cellulose), $a=1$, $\tau_{u b}=0.018 \pm 0.002 \mathrm{~cm}, \rho_{u b}=0.66 \pm 0.05 \mathrm{~g} / \mathrm{cm}^{3}$ and $\rho_{O 2}=1.376 \times 10^{-3} \mathrm{~g} / \mathrm{cm}^{3}$. The values $\tau_{b}=0.01 \pm 0.003 \mathrm{~cm}$ and $\rho_{b}=0.3 \pm 0.03 \mathrm{~g} / \mathrm{cm}^{3}$ are the result of averaging over 10 samples in the $V_{O 2}$ range of $0.5-15 \mathrm{~cm} / \mathrm{s}$. Both $\tau_{b}$ and $\rho_{b}$ increase in that range but do not change by more than $20 \%$.

[17] For a similar dependence of spacing between fingers on growth velocity in eutectic growth, see: V. Seetharaman and R. Trivedi, Metallurgical Transactions A 19A, 2956 (1988).

[18] We expect $P e_{c}$, as well as the characteristic length $w$, to be explicitly given by a linear stability analysis of the onset regime (which include heat transport). This study is in our current focus.

[19] We take pleasure in thanking J-P. Eckman, J. Fineberg, B. Matkowsky, G.I. Sivashinsky, V. Steinberg and L. Troyansky for stimulating discussions, and thank T. Kashiwagi for communicating results prior to publication. 


\section{FIGURES}

FIG. 1. Schematic representation of the setup. 1. Transparent top, 2. variable gap between top and bottom plates $(h) 3$. outflow of combustion products, 4 . spacers to control $h$, 5 . ignition wire (tungsten), 6. heat conducting boundaries (also serves to decrease the supply of oxygen from the sides of the sample), 7. flame front, 8. fuel (Whatman filter paper $\# 2,20 \times 20 \mathrm{~cm}^{2}$ ), 9. interchangeable bottom plate (to study of the role of the boundary's heat conductivity) 10 . uniform flow of $\mathrm{O}_{2} / \mathrm{N}_{2}$ (the uniformity was verified with smoke visualization), 11. gas diffuser, 12 . gas inlet.

FIG. 2. The instability as a function of oxygen flow $V_{O 2}$ (decreasing from $a$ to $e$ ). ( $a$ ) smooth front, $(b)$ irregular front, $(c)$ periodic pattern, $(d)$ fingering pattern with tip splitting, $(e)$ fingering without tip splitting. The pattern defines two lengths the finger width $w$ and spacing between fingers $d$. Both lengths stabilize within a short initial transient. The scale bars are $1 \mathrm{~cm}$. The gap between plates is $h=0.5 \mathrm{~cm}$. The oxygen flow velocity $V_{O 2}$ is directed downwards and the front velocity $u$ is directed upwards. The values are (top to bottom): $V_{O 2}=11.4,10.2,9.2,1.3,0.1 \mathrm{~cm} / \mathrm{s}$ and $u=0.5,0.48,0.41,0.14,0.035, \pm 0.01 \mathrm{~cm} / \mathrm{s}$.

FIG. 3. The schematic flow field. The flow lines are diverted by lateral diffusion which is driven by the deficiency of oxygen at the tips.

FIG. 4. Mass conservation, as predicted by Eq. 1 The insert shows $u$ as a function of $V_{O 2}$. The arrow corresponds to $P e_{c 1}$. We verified that Eq. 1 is also satisfied when the front propagates with the wind and when the parameter being varied is the fraction of oxygen in the feeding gas $(0.5 \leq a \leq 1)$. The oxygen molecular diffusion coefficient is taken to be $D=0.25 \mathrm{~cm}^{2} / \mathrm{s}$. We neglect its temperature dependence.

FIG. 5. The length scales $d$ (full circles) and $w$ (empty circles) as a function of $P e . d$ is determined by the driving parameter, while $w$ is weakly influenced by it. The slope of $w(P e)$ is $0.01 \pm 0.002 \mathrm{~cm}$ (dashed line). The continuous line is a plot of the RHS of It fits the measured $d$ with no adjustable parameter.

FIG. 6. The instability of an isolated finger $(w=0.5 \mathrm{~cm})$ is manifested as a periodic attempt to tip split in a thin paper of width $L=1 \mathrm{~cm}$ (bottom). It does not occur at $L=0.6 \mathrm{~cm}$. The distance between the two fingers was $10 \mathrm{~cm}$, ensuring no interaction between them $(h=0.5 \mathrm{~cm}, P e=6)$.

FIG. 7. The degree of two dimensionallity $(h)$ determines $w$ in a linear fashion. The slope is $1.78 \pm 0.4$. The data points are averages over a range of $V_{O 2}$ values. The spread is represented by the error bars. The empty circles at $h=0.5 \mathrm{~cm}$ are the data points of Fig. 


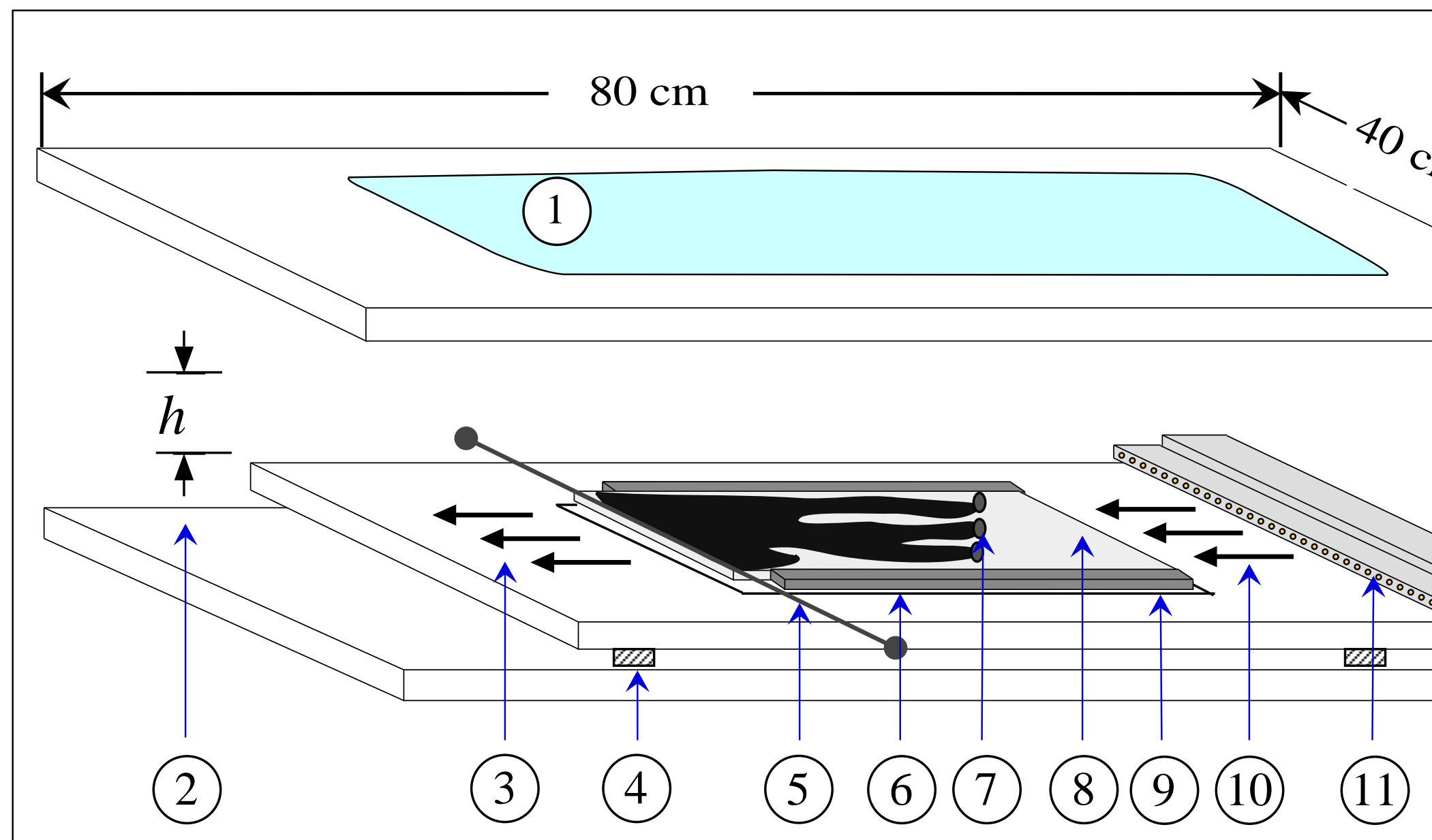




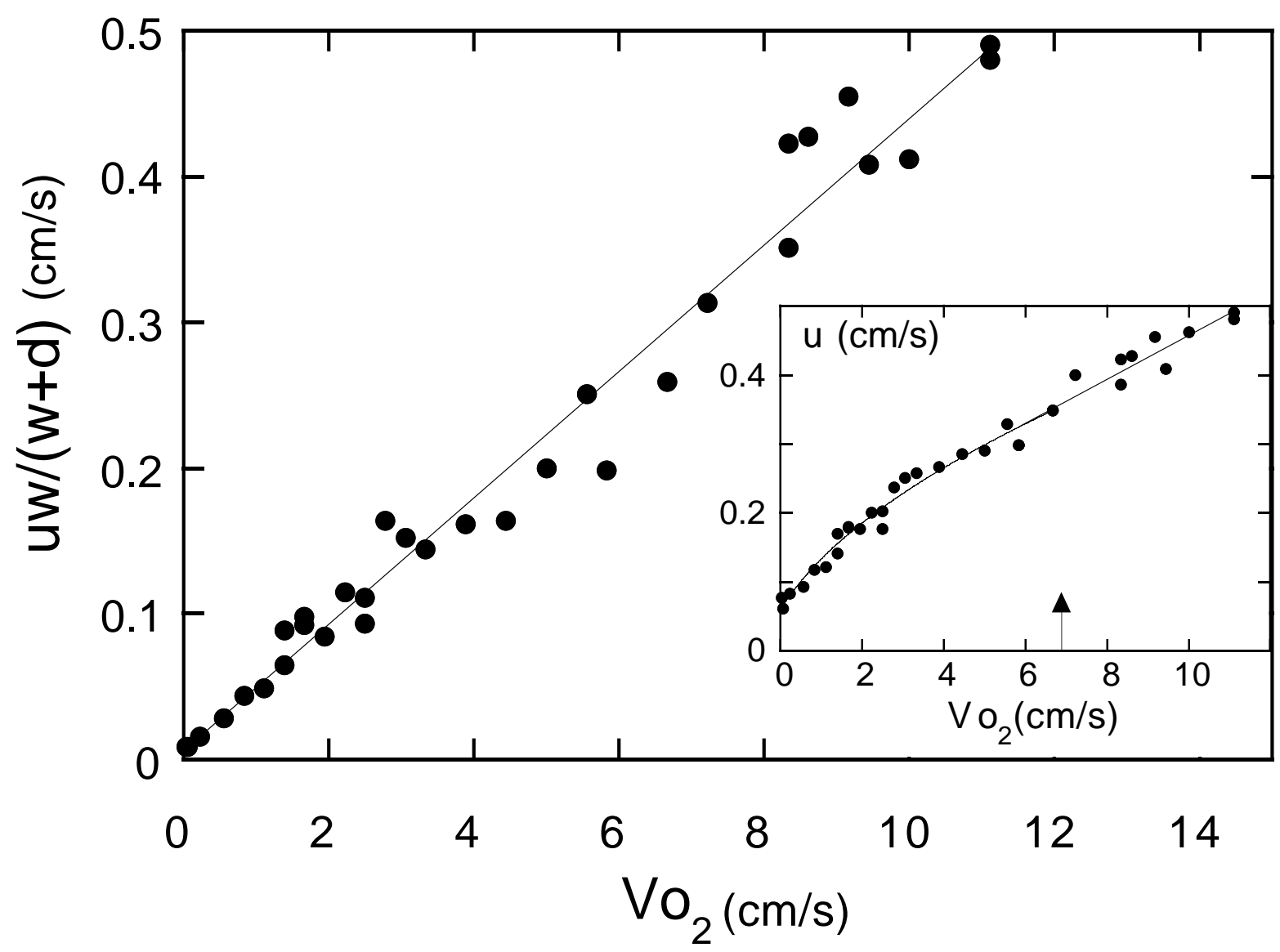




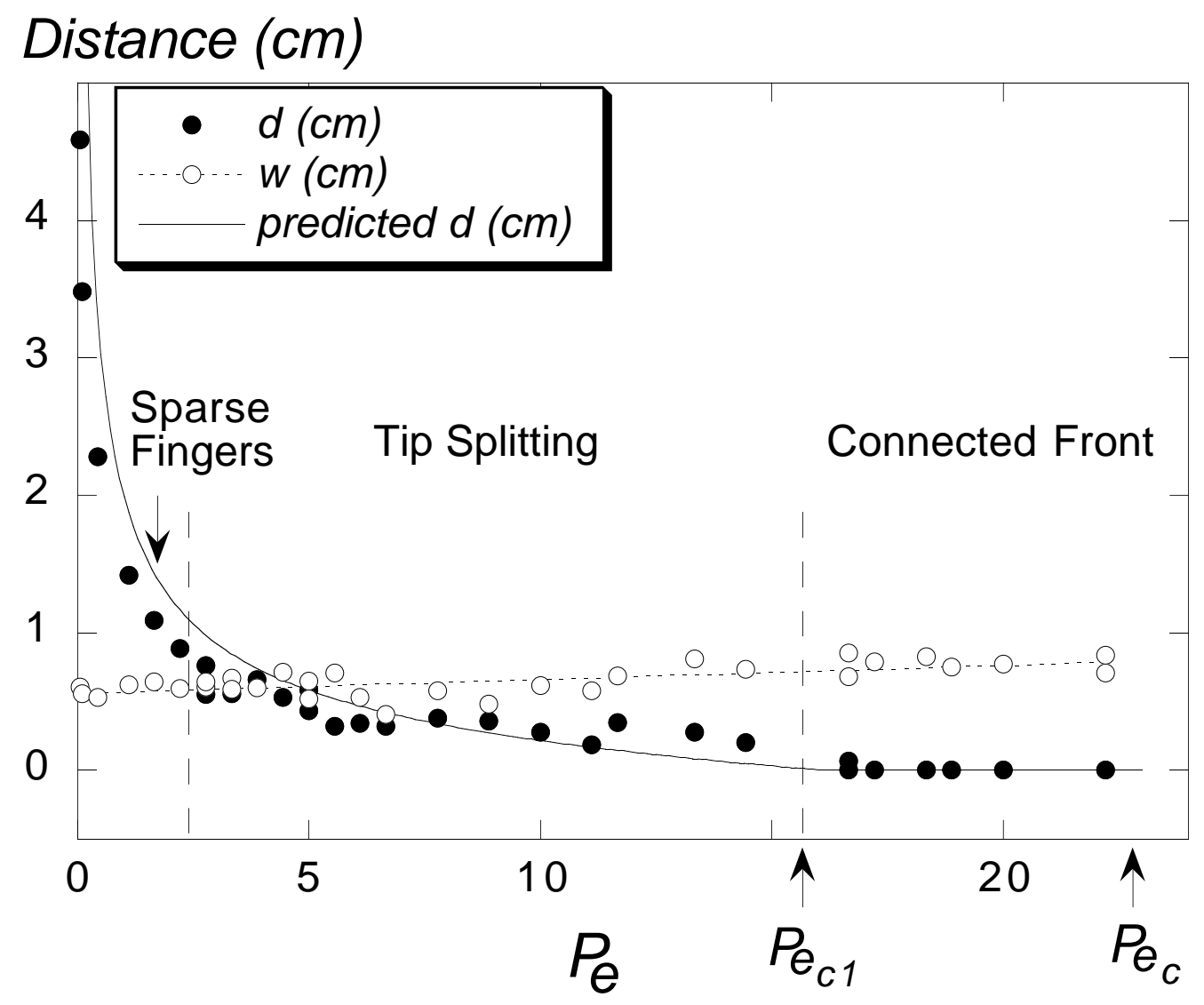


